\title{
Teenage monoarthralgia
}

Department of Clinical
Radiology, United
Bristol Healthcare
NHS Trust, Bristol,
United Kingdom
C J Wakeley
Department of
Radiology, Frenchay
Healthcare Trust,
Bristol, United
Kingdom
M J Cobby
Correspondence to:
Dr C J Wakeley,
Directorate of Clinical
Radiology, Marlborough
Street, Bristol BS2 8HW,
United Kingdom
^Department of Diagnostic
Imaging, The Robert Jones
and Agnes Hunt Orthopaedic
Hospital, Oswestry,
Shropshire SY10 7AG,
United Kingdom.

Radiology, United

NHS Trust, Bristol gdom

Radiology, French

Bristol, United

Radiogy, Marlborough

United Kingdom Shropshire SY10 7AG

United Kingdom.
Department of Clinical

Correspondence to:

\author{
Charles J Wakeley, Mark J Cobby
}

\section{Clinical history}

A 15 year old girl presented with a six month history of increasing pain and stiffness in her left hip. The pain was worse after exercise and she walked with a limp. On examination there was apparent shortening of the left lower limb with a ten degree fixed flexion deformity. Passive and active movements of the left hip were reduced in all directions and a positive Trendelenburg's sign was elicited. The full blood count, plasma viscosity, serum electrolytes, plasma proteins and urinalysis were all normal. Radiographs of the left hip were obtained and reported as normal (fig 1).

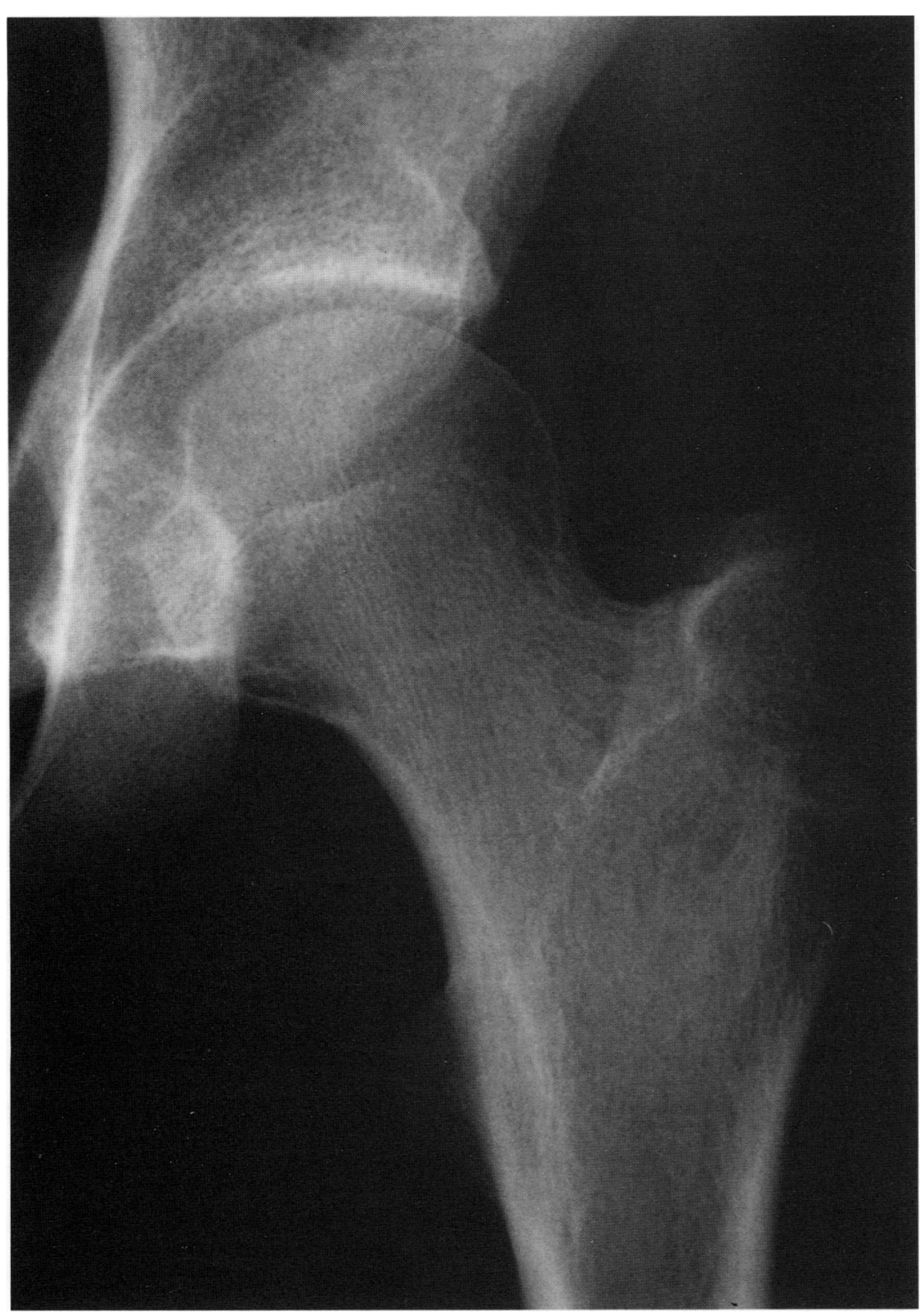

Figure 1 Anteroposterior radiograph of the left hip on presentation.
Conventional radiographs and MRI of the hip were obtained soon after admission (figs 1 and 2).

\section{Radiological findings}

The radiograph of the left hip at presentation was initially considered normal (fig 1). An MRI scan was then requested (fig 2 ), which clearly demonstrated a large zone of bone marrow oedema and a substantial joint effusion. Review of the conventional radiograph shows a radiolucent ring with a central zone of increased density (fig 3) and the diagnosis of an intra-articular osteoid osteoma was confirmed using thin section high resolution CT (fig 4). This elegantly demonstrated the osteoid osteoma with a calcified nidus within the anterior cortex, surrounded by a low attenuation uncalcified ring and a substantial joint effusion. Periosteal new bone formation was also evident extracapsularly along the anterior proximal femoral shaft.

\section{Differential diagnosis}

The MRI initially appear to be relatively nonspecific, leaving a wide differential diagnosis to be considered. This includes infection, infiltrative marrow disorders, an inflammatory synovitis, algodystrophy, transient bone marrow oedema and bone marrow contusion.

Once the radiolucent ring is identified on the conventional radiograph the diagnosis of an osteoid osteoma is virtually the only serious consideration and may be readily confirmed by radioisotope imaging or CT. The elegant demonstration by CT of the precise anatomical localisation of the nidus makes this the technique of choice.

\section{Diagnosis}

Intra-articular osteoid osteoma.

\section{Discussion}

The majority of osteoid osteomas are easily diagnosed from conventional radiographs and scintigraphy. However, intra-articular osteoid osteomas have an unusual radiological appearance that may lead to delay in their diagnosis and hence their treatment. This case study illustrates how such a diagnosis may be overlooked and, with the more frequent and early use of MRI, how non-specific the findings with this technique may be. 

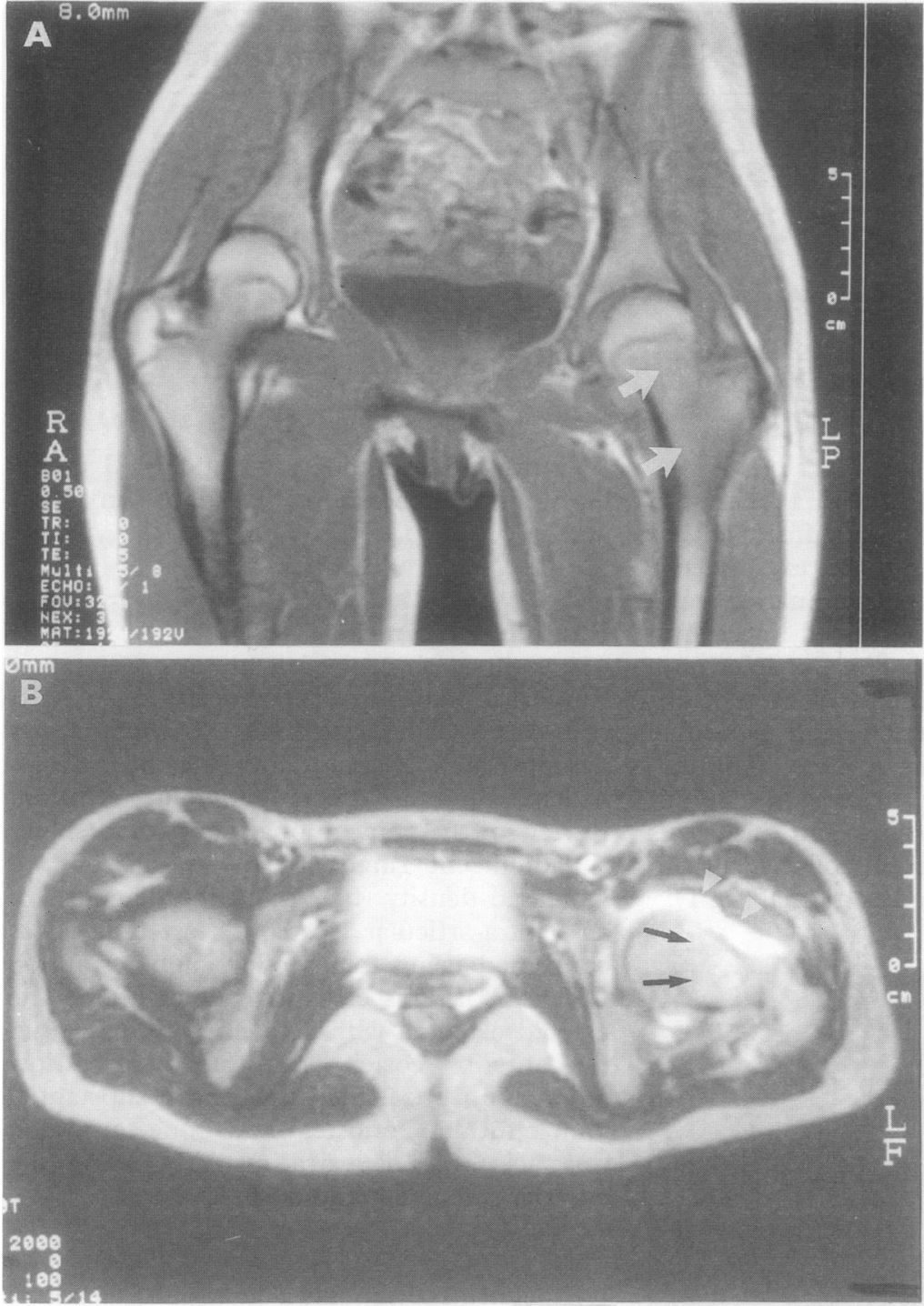

Figure 2 (A) Coronal T1-weighted (TR/TE 550/25) and (B) transaxial T2-weighted (TR/TE 2000/100) MRI through the hips. Bone marrow oedema is demonstrated in the left femoral neck and intertrochanteric region (arrows). This has a subtle low signal intensity on the T1-weighted image and a high signal intensity on the T2-weighted image. The margins to the abnormal area are poorly defined but do not involve the epiphysis. A substantial joint effusion is evident (arrow heads). No discrete focal abnormality is shown.

Twelve per cent of all benign bone tumours are osteoid osteomas. ${ }^{1}$ Several authors described the features of this benign tumour in the early 1930s, but it was Jaffe in $1935^{2}$ who introduced the term 'osteoid osteoma'. In 1966 Edeiken $^{3}$ classified three subtypes of osteoid osteoma, each with different radiological features and named according to their site of origin from the bone. The commonest subtype is the 'cortical' osteoid osteoma that typically arises in the cortex of a long bone. The tumour consists of a nidus of osteoid material (that may contain intra-tumoural calcification), and is surrounded by reactive peri-tumoural sclerosis. The 'cancellous' and 'subperiosteal' subtypes are less common. They usually arise in an intra-articular or juxta-articular position. Their radiological apjearances are less typical and more varied. Reactive osteosclerosis is often absent in this latter group and secondary features such as synovitis, a joint effusion and periosteal reaction may predominate. Even within these subtypes radiological features have been shown to vary between sites. ${ }^{4}$
This case is an example of an intra-articular osteoid osteoma. This rarer subtype of osteoid osteoma is particularly difficult to diagnose. In their case of osteoid osteoma of the talus, Yeager $e t a l^{5}$ failed to show any abnormality on the conventional radiograph. Likewise in this case the conventional radiographic findings were extremely subtle. It was the ability of the MRI to image the synovitis and altered marrow signal that prompted review of the conventional radiograph and suggested the diagnosis. However, MRI cannot yet distinguish between an inflammatory synovitis or infective synovitis from a synovitis secondary to an intraarticular osteoid osteoma. Indeed several authors warn of the danger in falsely interpreting the bone marrow changes as being secondary to either infection or a malignant neoplasm. ${ }^{6}$

A combination of factors may make the identification of an osteoid osteoma by MRI difficult. These include the relatively thick sections of the images compared with the size of the nidus, the frequent requirements of a slice interspace, and the poor ability of MRI to show small areas of calcification using conventional spin-echo techniques. In addition, peritumoural oedema or peritumoural sclerosis may be so overwhelming that it obscures the minute lesion (osteoid osteoma) causing it.

Treatment of an osteoid osteoma relies upon surgical excision of the nidus of the tumour. From reviewing previous reports it is evident that no single imaging modality will be diagnostic in every case of osteoid osteoma and it will often be necessary to use a combination of techniques to confirm the diagnosis of the exact size of its nidus.

Conventional radiography will demonstrate the nidus in most instances, but a tomographic technique may be required for accurate anatomical localisation. Kransdorf $e t a l^{7}$ found that the nidus was better demonstrated on CT than MRI in five of seven cases of osteoid osteoma and equally well in two cases. However, in one report MRI has been shown to be invaluable when the osteosclerotic reaction was so great that the nidus could not be seen on CT. ${ }^{7}$ Bone scintigraphy is readily available in most departments and the avid focal uptake on early images is characteristic. However, scintigraphy may be less specific when the lesion is intra-articular as the increase in activity can be generalised within the joint, due to associated synovitis, osteoporosis, and hyperaemia. ${ }^{8}$ Unfortunately, the clinical picture in patients with an intra-articular osteoid osteoma is also less typical. The classical nocturnal quality of pain found in $80 \%$ of extra-articular osteoid osteomas is absent and the pain is less responsive to salicylates. ${ }^{89}$

MRI signal characteristics are extremely varied for osteoid osteomas. ${ }^{5} 7$ 10-12 This is not surprising when the various subtypes and their differing plain radiographic features are considered. In general the osteoid nidus is depicted by a decrease in signal intensity on $\mathrm{T} 1$ weighted images and increased signal on T2 weighted images. Confusion in signal intensity 


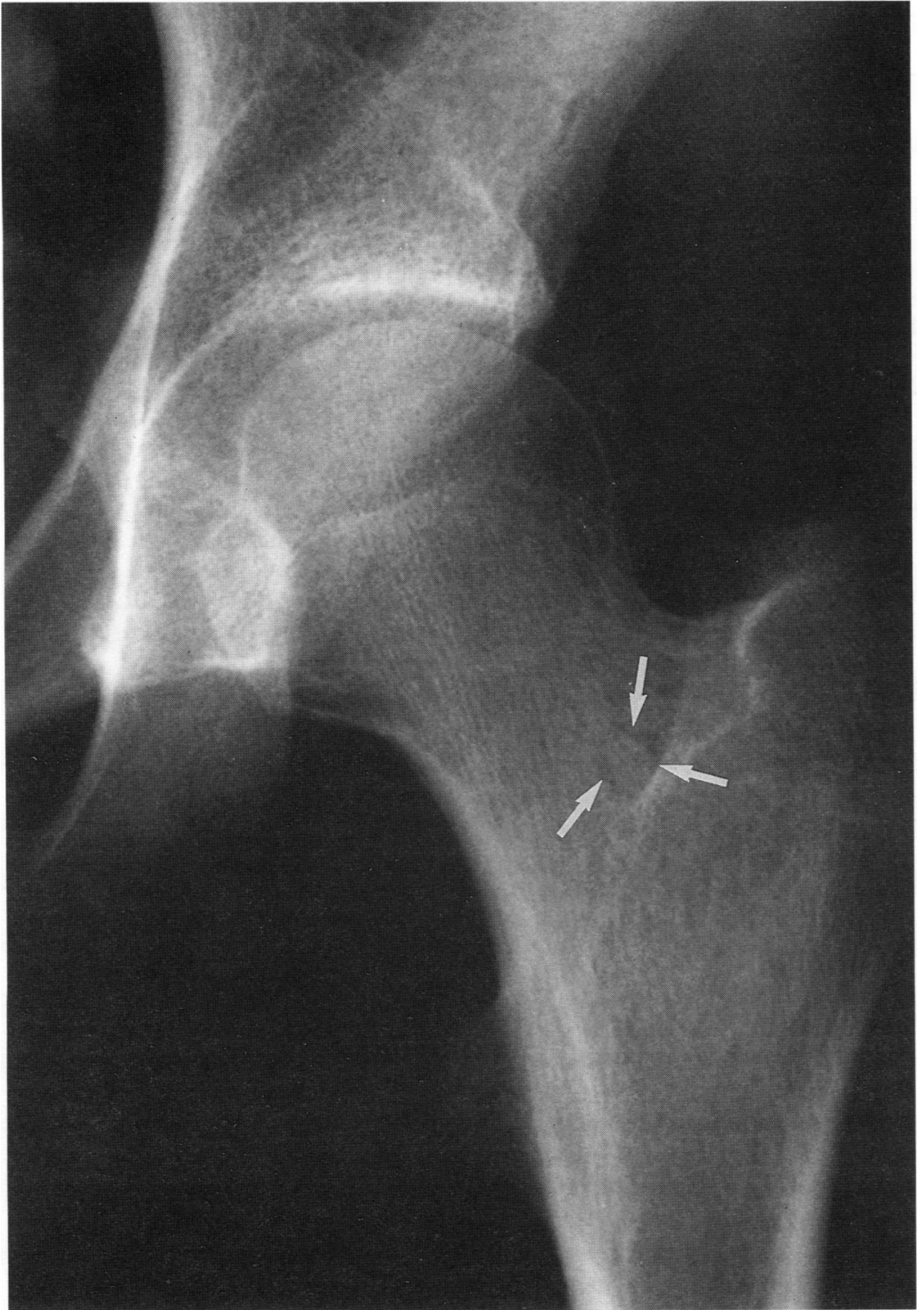

Figure 3 Same illustration as fig 1. The MRI examination prompted review of the conventional radiograph which was initially considered normal. A radiolucent ring, however, is evident in the femoral neck (arrows).

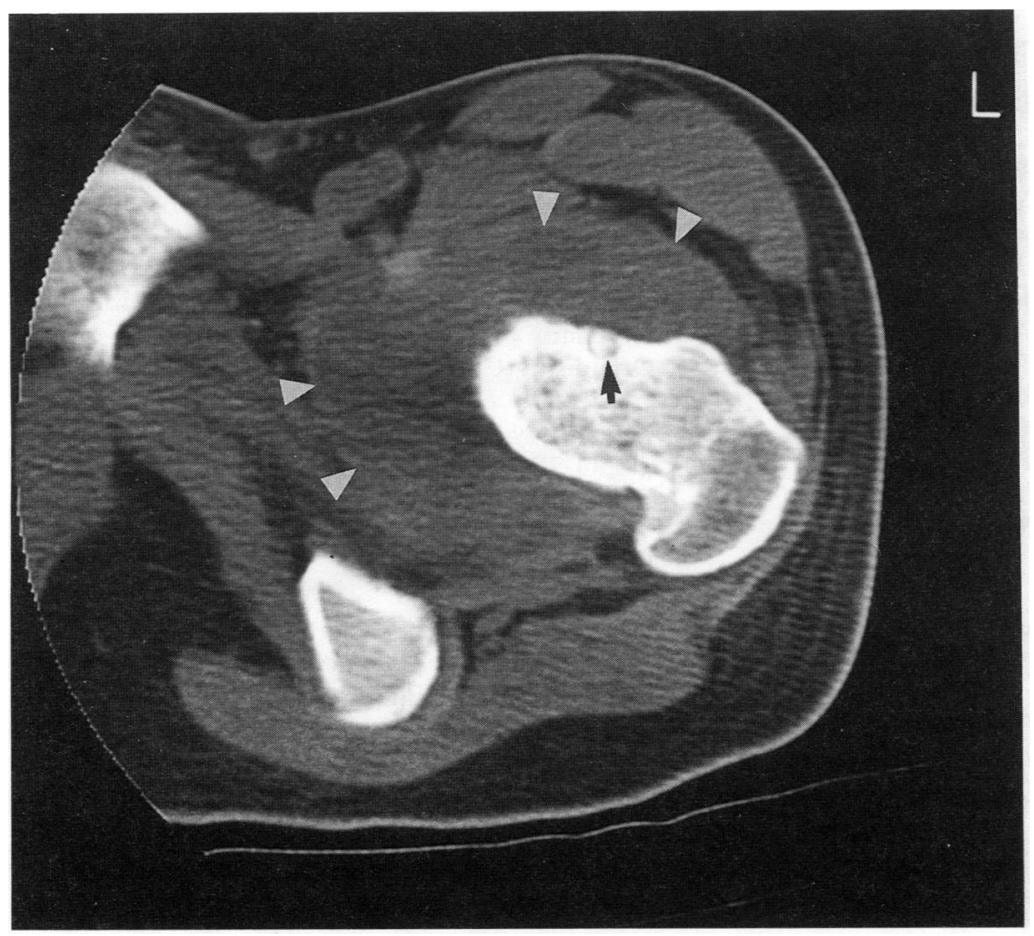

of the nidus has probably arisen due to varying amounts of intratumoural calcification which may alter these signal changes considerably. ${ }^{5} 12$ Adjacent to the osteoid nidus, surrounding sclerosis demonstrates a decrease in signal intensity, whereas surrounding oedema will show as an increase in signal intensity on T2 weighted sequences. The resulting signal characteristics again will differ according to the proportion of oedema and sclerosis. It may well be that a more specific diagnosis could have been revealed by obtaining thinner slices or using intravenous gadolinium in combination with fat suppression techniques.

The mean age of patients with osteoid osteomas in the South West England Bone Tumour Registry was 18.3 years (range 2-62 years). In this young age group it is particularly important to keep ionising radiation to a minimum. Partly for this reason, and partly because of the superb contrast resolution of MRI, it is likely that MRI will become a common primary or secondary imaging technique for many clinical problems in patients of this age group. Furthermore, the MRI examinations may well be performed and interpreted without access to other investigations. It is therefore important to be aware of the possible pitfalls of MRI in the diagnosis of an apparent monoarthritis.

In conclusion, this case study shows how the diagnosis of an intra-articular osteoid osteoma was suggested from apparently non-specific MRI appearances. It must be stressed, however, that no single imaging modality is always diagnostic in this condition and that the MRI appearances can actually be confusing, misleading, or may be falsely interpreted as infection or a malignant neoplasm.

1 Dahlin D C, Unni K K. Bone tumors: general aspects and data on 8542 cases, 4th ed. Springfield, ill: Thomas, 1987: on 8542

2 Jaffe H L. Osteoid osteoma: a benign osteoblastic tumor composed of osteoid and atypical bone. Arch Surg 1935; 31: 709-28.

3 Edeiken J, DePalma A F, Hodges P J. Osteoid osteoma (roentgenographic emphasis). Clin Orthrop 1966; 49: 201-6.

4 Moser R P, Kransdorf M J, Brower A C, et al. Osteoid osteoma of the elbow. Skeletal Radiol 1990; 19: 181-6.

5 Yeager B A, Schiebler M L, Wertheim S B, et al. Case report: MR imaging of osteoid osteoma. f Comput Assist Tomogr 1987; 11: 916-7.

6 Assoun J, Richardi G, Railhac J J, Baunin C, Bonnevialle P. Osteoid osteoma: MR imaging versus CT. RSNA 79th Scientific assembly and annual meeting 1993.

7 Kransdorf M J, Stull M A, Gilkey F W, Moser R P. Osteoid osteoma. Radiographics 1991; 11: 671-96.

8 Cassar-Pullicino V N, McCall I W, Wan S. Intra-articular osteoid osteoma. Clinical Radiology 1992; 45: 153-60.

9 Cohen M D, Harrington T M, Ginsburg W W. Osteoid osteoma: 95 cases and a review of the literature. Seminars in arthritis and Rheumatism 1983; 12: 265-81.

10 Bell R S, O'Connor G D, Wadell J P. Importance of magnetic resonance imaging in osteoid osteoma: A case report. Can $\mathcal{F}$ Surg 1989; 32(4): 276-8.

11 Glass R B J, Poznanski A K, Fisher M R, Shkolnik A, Dias L. Case report. MR imaging of osteoid osteoma. 7 Comput Assist Tomogr 1986; 10: 1065-67.

12 Houang B, Grenier N, Greselle J F, et al. Osteoid osteoma of the cervical spine: misleading MR features about a case
involving the uncinate process. Neuroradiolgy 1990; 31: 549-51.

Figure 4 Transaxial high resolution CT section through the left femoral neck. $A$ densely calcified nidus within the anterior cortex of the intra-articular portion of the femoral neck is demonstrated (arrow) and surrounded by a well defined radiolucent ring. $A$ substantial joint effusion is present (arrow heads). The appearances are characteristic of an osteoid osteoma with a reactive synovitis. 